\title{
SELF-INSURANCE OF INVESTOR UNDER REPEATING CATASTROPHIC RISKS ${ }^{1}$
}

\author{
V. I. Norkin
}

UDC 519.218

\begin{abstract}
A decision-making problem of investment into a profitable object in a catastrophic risk area is considered. By a catastrophic risk is meant the probability of severe yet unlikely losses. As a risk hedging mechanism, an insurance fund is considered that is replenished by a part of profit and is used for object renewal. It is shown that methods of insurance mathematics can be used to assess the risk to lose the object. For the plant loss probability as a function of the insurance reserve, integral equations are derived. They can be solved by successive approximations.
\end{abstract}

Keywords: investments, catastrophic risk, insurance, risk estimate, risk measure, random risk process, financial mathematics, actuarial mathematics, loss probability, integral equations, method of successive approximations.

\section{INTRODUCTION}

The study is concerned with quantitative estimation of the risk of investment decisions. An elementary binary decision-making problem is considered. It deals with investing in a quite profitable object in an area of high or catastrophic risk (for example, with repeating catastrophic flood, tsunami, etc.). By a catastrophic risk is meant the probability of severe yet unlikely losses. Despite the apparent simplicity of the problem, the solution is not always obvious and requires careful simulation and risk analysis.

The difficulty is that this seemingly simple solution should be based on profit-risk analysis and may contain additional parameters. The investor should examine several candidate solutions different in profit and risk to choose the most appropriate one. If, for example, the investor partially insures the investments, then the investment decision will also include a decision on the level of insurance, thus becoming dependent on insurance rates and other insurance conditions. However, insurance companies do not always agree to insure catastrophic risks [1] because of the difficulty of assessing them. In this case, the investor may use self-insurance by creating an initial insurance fund and then supplementing it by a part of profit. An object of investments suffered from a catastrophic event should be recovered at the expense of the insurance fund. Catastrophic events and restoration of the object may repeat. In this case, the investment decision also includes a decision on the level of the initial insurance fund and the amount of annual profit contribution to the insurance fund. There may be two situations (depending on the level of the profit contribution):

(i) the object of investments is lost sooner or later (with unit probability) after it earns a sufficient profit;

(ii) the object functions virtually infinitely long with positive probability and, therefore, earns a constant profit for an infinitely long time.

In what follows, we will mainly analyze the second situation. To this end, we adapt the Cramer-Lundberg risk theory, in particular, the theory of actuarial integral equations. The analytic analysis of the first situation is more difficult since it is necessary to find the distribution of the cumulative profit obtained prior to a random moment the object is lost. However, both situations can be analyzed numerically by statistical modeling (Monte-Carlo method). It is also possible to sell the object constructed. An additional task is to choose the optimal moment for the sale (shutdown).

${ }^{1}$ The study was sponsored by the Ukrainian Scientific and Technical Center (UNTTs, Project G3127)

V. M. Glushkov Institute of Cybernetics, National Academy of Sciences of Ukraine, Kyiv, Ukraine, norkin@i.com.ua. Translated from Kibernetika i Sistemnyi Analiz, No. 3, pp. 74-83, May-June 2007. Original article submitted August 7, 2006. 
The objects located in a risk-free area and available for the investor may also be an insurance. In case of a catastrophic event, a destroyed object is restored at the expense of the profit from unaffected objects. Then there arises the problem of rational investment in risk areas or the problem of optimal investment portfolio under catastrophic risks. There is an extensive portfolio theory of investments [2] based on the variance of profit as the basic risk measure for the investor. However, this theory is hardly applicable under catastrophic risks since the variance is not an adequate measure of risk in this case. Other risk measures, defined on the tail of loss distribution [3] can be used in this case. In particular, the idea of profiling single catastrophic risks as risk control means is employed in [4].

Investments in high-risk areas (river or ocean coast, valley or high-water bed, bottom of a volcano, etc.) are attractive since they are often very profitable due to low construction cost and high return from investment. However, the object of investments may be partially destroyed because of a catastrophic event and may require (repeatedly) additional investments for its restoration and further profitable operation. Additional investments can arrive from a special insurance fund, from other risk-free sources of income or from a credit. And if there are no reserves for the restoration of the object, initial investments may be lost irretrievably. Thus, the emergency fund is a means of self-insurance of the investor. The problem of formal analysis (the main purpose of this paper) consists in estimating the risk (probability) of an irretrievable loss of investments. The main result of the present paper is that the Cramer-Lundberg model of collective risk [5-7], widely used in actuarial (insurance) mathematics to simulate the stochastic evolution of the capital of an insurance company, can also be used to simulate the individual risk of an investor. This makes it possible to apply classical and modern insurance mathematics to estimate the investor's risk. The probability of irretrievable loss of business (investee) is used as a measure of risk of the investor. Integral equations for (non)loss-of-business probability as a function of the initial insurance reserve will be derived in the paper. We will detail a model with discrete time and a finite set of catastrophic scenarios. In this case, the integral equation for the probability of (non-) loss of business is transformed to a functional-difference equation. The sufficient existence and uniqueness conditions of its solution will be established. The use of the method of successive approximations is justified and the exponential lower bound of the solution is determined. The results are illustrated with a numerical example.

\section{CLASSICAL DYNAMIC MODEL OF INVESTMENT DECISIONS: A RISK-RESTORATION PROCESS}

Assume that time of spending investments (construction of the object) is short compared with the average period between catastrophic events, for example, between repeated flood. The mathematical model is based on the assumption that investments are made instantaneously. Denote by $v$ the cost of the object of investments and by $u$ the capital remaining after the construction (insurance fund). The basic variable in the model is the amount of the emergency fund $R_{t}$, where the index $t$ denotes the current time. The insurance fund is changed as follows. The object constructed brings in a constant return per unit time, which partially (in the amount $c$ ) replenishes the insurance fund. At a random time, a catastrophic event happens and partially destroys the object. Object restoration is paid for from the insurance fund, which thus decreases, by the value of damage. The object restored again brings in a return whose part replenishes the insurance fund. However, if the insurance fund is insufficient at the time of catastrophe to restore the object, the latter is lost irretrievably and, thus, the initial investments $v$ are lost irretrievably. Assume that the cost of the object after catastrophe decreases and equals $z v$, where $z$ is a random variable, $0 \leq z \leq 1$. Denote by $F(\cdot)$ the cumulative distribution function of the random variable $z$. Let $N_{t}$ be the number of catastrophic events before the moment $t$, and $z_{k}$ be a coefficient of object damage at the time of the $k$ th catastrophe. Then the dynamics of the insurance fund can be described by the equation

$$
R_{t}=u+c t-\sum_{k=1}^{N_{t}} z_{k} v, \quad t \geq 0 .
$$

This classical equation for a risk process in actuarial mathematics describes the dynamics of the capital of an insurance company that accumulates a great number of independent risks [5, Eq. (3.6)]. If $R_{t}$ becomes less than zero at any time, the company is bankrupted. In our model, such an event means a retrievable loss of initial investments. We are interested in the probability of losing (or returning) initial investments. We will consider it as a function of the initial insurance fund $u$. Let time intervals between sequential catastrophes be distributed according to the cumulative distribution function $K(t)$, and $t$ be the time passed from the last catastrophe before the construction of the object. The 
nonbankruptcy probability $\varphi(u)$ of the risk process (in our case, the probability not to lose the initial investments) is known to be calculated as follows [5, Eq. (3.72)]:

$$
\varphi(u)=\int_{0}^{+\infty} \int_{0}^{\min \{1,(u+c t) / v\}} \varphi_{0}(u+c \tau-z v) d F(z) d K_{t}(\tau),
$$

where $\varphi_{0}(\cdot)$ satisfies the following integral equation (ordinary renewal equation) [5, Eq. (3.74)]:

$$
\varphi_{0}(u)=\int_{0}^{+\infty} \int_{0}^{\min \{1,(u+c t) / v\}} \varphi_{0}(u+c \tau-z v) d F(z) d K(t) ;
$$

$K_{t}(\tau)=[K(t+\tau)-K(\tau)] /[1-K(t)]$ is the cumulative distribution function for the moment of the first catastrophic event after object construction, provided that the time $t$ has passed from the last catastrophic event before the construction.

Note that the solution $\varphi_{0}(u)$ of the linear homogeneous integral equation (3) with the infinite integration domain must satisfy the boundary condition at infinity

$$
\varphi_{0}(+\infty)=\lim _{u \rightarrow+\infty} \varphi(u)=1 .
$$

If the cumulative distribution function $K(t)$ for time intervals between sequential catastrophes is exponential, i.e., $K(t)=1-e^{-\alpha t}$, and the flow of catastrophic events is Poison, then Eqs. (2) and (3) with the boundary condition (4) can be reduced to the Volterra integral equation for nonbankruptcy probability [5, Eq. (3.13)]:

$$
\varphi(u)=1-\frac{\alpha \mu}{c}+\frac{\alpha}{c} \int_{0}^{u / v} \varphi(u-z v)[1-F(z)] d z
$$

where $\mu$ is the mean damage from catastrophes, $\mu=v \int_{0}^{1}(1-F(z)) d z$. There is an extensive theory and various analytic and numerical methods to solve Eq. (5) [6, 7]. To solve Eq. (3) with the boundary condition (4), the method of successive approximations is justified in [8].

\section{MODEL WITH DISCRETE TIME}

In some applications, repeating catastrophes (for example, catastrophic flood) are modeled as follows. A catastrophe of certain strength $\omega$ at a random time $\tau, 0 \leq \tau \leq 1$, is assumed to be possible during a time unit (for example, a year or another economic-climatic cycle) with a certain probability described by the cumulative distribution function $F(\omega, \tau)$. For example, in case of catastrophic flood, the parameter $\omega$ describes a catastrophic water flow rate in an upper section of the river channel. We also assume that catastrophes are rare events, i.e., we neglect the possibilities of two catastrophes during one time unit. Let $z_{\omega, \tau}$ be a random portion of the object cost lost in a catastrophic event $\omega$ occurred at the time $\tau$, and $y_{\omega, \tau}$ denote a random portion of the target profit obtained due to such a catastrophic event. By definition, $0 \leq z_{\omega, \tau} \leq 1$, $0 \leq y_{\omega, \tau} \leq 1$. For example, to determine the value of $z_{\omega, \tau}$ in case of a catastrophic flood of strength $\omega$, a flood wave passing through the river channel is modeled, maps of maximum flooding are designed on this basis, and the flood level and duration for a specific object are found. Then empirical curves (moment, level, and duration of flood - percentage damage) are used to find the distribution of the relative damage $G_{\omega, \tau}(z)$. The distribution $H_{\omega, \tau}(y)$ of the portion of the profit lost as a result of the catastrophe $(\omega, \tau)$ is constructed similarly based on the description of the profit earning process.

As before, denote by $v$ the value of the object of investment, by $u$ the initial amount of the emergency fund; by $c$ the income per unit time due to object functioning that arrives at the emergency fund; by $z_{\omega, \tau} v$ random loss in the object cost as a result of the catastrophe $\omega$; and by $y_{\omega, \tau} c$ random loss of profit. The dynamics of the emergency fund can be described similarly to Eq. (1):

$$
R_{t+1}=R_{t}+c-\sum_{k=1}^{N_{t}} y_{k} c-\sum_{k=1}^{N_{t}} z_{k} v, R_{0}=u, t=0,1, \ldots,
$$

where $y_{k}$ and $z_{k}$ are loss factors of profit and of the object cost as a result of the $k$ th catastrophic event. 
Let $\varphi(u)$ be the probability of returning the investments with the initial insurance fund $u$. This probability satisfies the integral equation (derived similarly to Eq. (3.12) from [5])

$$
\varphi(u)=\int_{\Omega \times[0,1]} \int_{0}^{(u+c) / c} \int_{0}^{(u+c-y c) / v} \varphi(u+c-y c-z v) d G_{\omega, \tau}(z) d H_{\omega, \tau}(y) d F(\omega, \tau)
$$

with the boundary condition at infinity $\varphi(+\infty)=1$.

Similar models and equations (with random profit) and some methods of their solution are considered in $[9,10]$.

\section{MODEL WITH A DISCRETE SET OF CATASTROPHIC SCENARIOS}

In applications, a discrete set of catastrophic scenarios $\omega_{1}, \omega_{2}, \ldots, \omega_{N}$ with probabilities $p_{1}, p_{2}, \ldots, p_{N}$ are often considered. We simply denote the corresponding relative loss by $z_{1}, z_{2}, \ldots, z_{N}$, and the loss distributions in these scenarios by $G_{1}(z), G_{2}(z), \ldots, G_{N}(z)$. Denote by $\omega_{0}$ (the unique) non-catastrophic scenario that is implemented with (a high) probability $p_{0}=1-\sum_{n=1}^{N} p_{N}$. There is no damage for the object of investments in this case, hence it earns a complete income $c$, i.e., $z_{0} \equiv 0, y_{0} \equiv 0$. Let, in case of any catastrophic scenario implemented, the object earn no income at all, i.e., then $y_{k} \equiv 1$. Then Eq. (6) becomes

$$
\varphi(u)=p_{0} \varphi(u+c)+\sum_{n=1}^{N} p_{n} \int_{0}^{u / v} \varphi\left(u-z_{n} v\right) d G_{n}\left(z_{n}\right) .
$$

If the loss $z_{n}$ as a result of the catastrophic scenario $n$ are deterministic, then Eq. (7) becomes simple:

$$
\varphi(u)=p_{0} \varphi(u+c)+\sum_{z_{n} \leq u / v} p_{n} \varphi\left(u-z_{n} v\right) .
$$

Remark. The investment decision in the model considered depends on the profit-loss set $(c, z)$. A risk measure for such a decision is the probability $\varphi(u)$, which also depends on $c, z=\left(z_{1}, \ldots, z_{N}\right), \varphi(u)=\varphi_{c, z}(u)$. The probability $\varphi(u)$ is a non-monetary risk measure. We can introduce a monetary risk measure (of level $\alpha$ )

$$
r_{\alpha}(c, z)=\min \left\{u \geq 0: \varphi_{c, z}(u) \geq 1-\alpha\right\}
$$

as the amount of the initial insurance fund that guarantees a certain probability of returning the investments. If the function $\varphi_{c, z}(u)$ is quasiconcave on the set of variables $(u, c, z)$, the risk measure $r_{\alpha}(c, z)$ is a function convex in $(c, z)$ since its epigraph $\left\{(u, c, z) \geq 0: \varphi_{c, z}(u) \geq 1-\alpha\right\}$ is a convex set in this case.

Let us use the approach developed in $[8,10,11]$ to analyze Eq. (8). Define the function

$$
\varphi_{*}(u)=1-e^{-\alpha u}
$$

where $\alpha$ is a positive root of the equation

$$
1-p_{0} e^{-\alpha c}=\sum_{n=1}^{N} p_{n} e^{\alpha z_{n} v}
$$

LEMMA 1. A positive root of Eq. (10) certainly exists if the mean profit due to investments is greater than the mean loss as a result of catastrophic events, i.e.,

$$
c>\frac{v}{p_{0}} \sum_{n=1}^{N} p_{n} z_{n} .
$$

Proof. The function $1-p_{0} e^{-\alpha c}=f_{l}(\alpha)$ from the left-hand side of Eq. (10) is concave in $\alpha$, monotonically increases to unity as $\alpha \rightarrow+\infty$, and monotonically decreases to minus infinity as $\alpha \rightarrow-\infty$. The function $f_{r}(\alpha)=\sum_{n=1}^{N} p_{n} e^{\alpha z_{n} v}$ is convex in $\alpha$, monotonically increases to plus infinity as $\alpha \rightarrow+\infty$, and monotonically decreases to zero as $\alpha \rightarrow-\infty$. For 
$\alpha=0$, the values of the right- and left-hand sides of Eq. (10) coincide: $f_{l}(0)=f_{r}(0)$. Therefore, a positive solution of Eq. (10) exists only if the relation $f_{l}^{\prime}(0)>f_{r}^{\prime}(0)$ holds for the derivatives of these functions at zero. Hence, condition (11) follows.

Let us consider an operator $A$,

$$
A \varphi(u)=p_{0} \varphi(u+c)+\sum_{z_{n} \leq u / v} p_{n} \varphi\left(u-z_{n} v\right),
$$

defined on bounded functions $\varphi(u), u \geq 0$, with the norm $\|\varphi\|=\sup _{u \geq 0}|\varphi(u)|$.

LEMMA 2. The operator $A$ is monotonic, i.e., for any bounded functions $\varphi_{1}$ and $\varphi_{2}$ such that $\varphi_{1}(u) \leq \varphi_{2}(u)$ for any $u \geq 0$, the inequality $A \varphi_{1}(u) \leq A \varphi_{2}(u)$ holds for any $u \geq 0$. Moreover, $A \varphi_{*}(u) \geq \varphi_{*}(u)$ and $A \mathbf{1}(u) \leq 1$, where $\mathbf{1}$ denotes the function identically equal to unity.

Proof. The monotonicity of the operator $A$ is obvious and follows from the positiveness of the probabilities $p_{0}, p_{1}, \ldots, p_{N}$. The following estimates are true:

$$
A \mathbf{1}(u) \leq p_{0}+\sum_{z_{n} \leq u / v} p_{n} \leq p_{0}+\sum_{n=1}^{N} p_{n}=1
$$

Let us show that $A \varphi_{*}(u) \geq \varphi_{*}(u)$ :

$$
\begin{gathered}
A \varphi_{*}(u)=p_{0}\left(1-e^{-\alpha(u+c)}\right)+\sum_{z_{n} \leq u / v} p_{n}\left(1-e^{-\alpha\left(u-z_{n} v\right)}\right) \\
=p_{0}\left(1-e^{-\alpha(u+c)}\right)+\sum_{n} p_{n}\left(1-e^{-\alpha\left(u-z_{n} v\right)}\right)-\sum_{z_{n}>u / v} p_{n}\left(1-e^{-\alpha\left(u-z_{n} v\right)}\right) \\
=1-e^{-\alpha u}\left(p_{0} e^{-\alpha c}+\sum_{n} p_{n} e^{\alpha z_{n} v}\right)+\sum_{z_{n}>u / v} p_{n}\left(e^{\alpha v\left(z_{n}-u / v\right)}-1\right) \geq 1-e^{-\alpha u}=\varphi_{*}(u) .
\end{gathered}
$$

The lemma is proved.

THEOREM 1 (existence and uniqueness of the solution of Eq. (8)). Given (11), Eq. (8) has a unique monotonic (nondecreasing) solution $\varphi(u)$ continuous on the right and such that $\varphi_{*}(u) \leq \varphi(u) \leq 1$.

Proof. Let us consider a sequence of approximations $\varphi^{k+1}(u)=A \varphi^{k}(u), \varphi^{0}(u) \equiv 1, k=0,1, \ldots$. By virtue of the property $A \mathbf{1} \leq 1$, the inequality $\varphi^{1}(u)=A \mathbf{1} \leq 1=\varphi^{0}(u)$ holds. Herefrom, by virtue of the monotonicity of the operator $A$ it follows that $\varphi^{k+1}(u) \leq \varphi^{k}(u) \leq 1$ for all $k$, i.e., the sequence $\left\{\varphi^{k}(u)\right\}$ monotonically decreases.

All the functions $\varphi^{k}(u)$ are monotonic (do not decrease) in $u$. Indeed, if $\varphi^{k-1}(u)$ is a function nondecreasing in $u$, then

$$
\begin{gathered}
\varphi^{k}\left(u_{1}\right)=A \varphi^{k-1}\left(u_{1}\right)=p_{0} \varphi^{k-1}\left(u_{1}+c\right)+\sum_{z_{n} \leq u_{1} / v} p_{n} \varphi^{k-1}\left(u_{1}-z_{n} v\right) \\
\leq p_{0} \varphi^{k-1}\left(u_{2}+c\right)+\sum_{z_{n} \leq u_{2} / v} p_{n} \varphi^{k-1}\left(u_{2}-z_{n} v\right)=A \varphi^{k-1}\left(u_{2}\right)=\varphi^{k}\left(u_{2}\right)
\end{gathered}
$$

for any $u_{1}<u_{2}$. Since the initial function is monotonic in $u$, all the approximations are monotonic in $u$ as well.

All the functions $\varphi^{k}(u)$ are continuous on the right in $u$. Indeed, let $\varphi^{k-1}(u)$ be a function continuous on the right, i.e., $\lim _{m \rightarrow \infty} \varphi^{k-1}\left(u_{m}\right)=\varphi^{k-1}(u)$ for any sequence $\left\{u \leq u_{m} \rightarrow u\right\}$. Without loss of generality, we may assume that $\varphi^{k-1}(u)=0$ for $u<0$. Then

$$
\lim _{m \rightarrow \infty} \varphi^{k}\left(u_{m}\right)=\lim _{m \rightarrow \infty}\left(p_{0} \varphi^{k-1}\left(u_{m}+c\right)+\sum_{z_{n} \leq u_{m} / v} p_{n} \varphi^{k-1}\left(u_{m}-z_{n} v\right)\right)
$$




$$
\begin{gathered}
=\lim _{m \rightarrow \infty}\left(p_{0} \varphi^{k-1}\left(u_{m}+c\right)+\sum_{n} p_{n} \varphi^{k-1}\left(u_{m}-z_{n} v\right)\right) \\
=p_{0} \lim _{m \rightarrow \infty} \varphi^{k-1}\left(u_{m}+c\right)+\sum_{n} p_{n} \lim _{m \rightarrow \infty} \varphi^{k-1}\left(u_{m}-z_{n} v\right)=p_{0} \varphi^{k-1}(u+c)+\sum_{n} p_{n} \varphi^{k-1}\left(u-z_{n} v\right)=\varphi^{k}(u) .
\end{gathered}
$$

Since the initial function is continuous on the right in $u$, all the approximations are continuous on the right in $u$ as well.

Since $\varphi_{*}(u) \leq 1$, from the monotonicity of the operator $A$ and the property $\varphi_{*}(u) \leq A \varphi_{*}(u)$ it follows that $\varphi_{*}(u) \leq \varphi^{k}(u)$ for all $k$. Thus, the sequence $\left\{\varphi^{k}(u)\right\}$ converges pointwise to a monotonic function $\varphi(u)$ such that $\varphi_{*}(u) \leq \varphi(u) \leq 1$. Passing to the limit with respect to $k$ in the equality $\varphi^{k+1}(u)=A \varphi^{k}(u)$, we get $\varphi(u)=A \varphi(u)$, i.e., the function $\varphi(u)$ satisfies Eq. (8).

Let us show that the function $\varphi(u)$ is continuous on the right. Let $\left\{u \leq u_{m} \rightarrow u\right\}$. Since we have proved that the function $\varphi$ and the sequence $\left\{\varphi^{k}\right\}$ are monotonic, the inequalities $\varphi(u) \leq \varphi\left(u_{m}\right) \leq \varphi^{k}\left(u_{m}\right)$ hold. Passing to the limit with respect to $m$ in these inequalities, we get

$$
\varphi(u) \leq \lim _{m} \varphi\left(u_{m}\right) \leq \lim _{m} \varphi^{k}\left(u_{m}\right)=\varphi^{k}(u) .
$$

With $\lim _{k} \varphi^{k}(u)=\varphi(u)$, it follows that $\lim _{m} \varphi\left(u_{m}\right)=\varphi(u)$, i.e., the function $\varphi(u)$ is continuous on the right. Thus, we have proved the existence of a continuous solution nondecreasing on the right.

Let us prove the uniqueness of the solution. Let $\varphi_{1}(u)$ and $\varphi_{2}(u)$ be two different solutions of Eq. (8) such that $\varphi_{*}(u) \leq \varphi_{1}(u) \leq 1$ and $\varphi_{*}(u) \leq \varphi_{2}(u) \leq 1$. Obviously, $\left|\varphi_{1}(u)-\varphi_{2}(u)\right| \leq 1-\varphi_{*}(u)$. Let

$$
|| \varphi_{1}-\varphi_{2} \|=\lim _{k \rightarrow \infty}\left|\varphi_{1}\left(u^{k}\right)-\varphi_{2}\left(u^{k}\right)\right| \geq \varepsilon>0 .
$$

Since $\left|\varphi_{1}\left(u^{k}\right)-\varphi_{2}\left(u^{k}\right)\right| \leq 1-\varphi_{*}\left(u^{k}\right)$ and $\lim _{u \rightarrow \infty}\left(1-\varphi_{*}(u)\right)=0$, the sequence $\left\{u^{k}\right\}$ is bounded. Let $\bar{u}$ be the maximum limiting point of the numerical sequence $\left\{u^{k}\right\}$; without loss of generality, we may assume that $\lim _{k \rightarrow \infty} u^{k}=\bar{u}$. Then for any $\varepsilon<c$ there exists a positive $\delta$ such that $\left|\varphi_{1}(u)-\varphi_{2}(u)\right| \leq|| \varphi_{1}-\varphi_{2} \|-\delta$ for all $u \geq \bar{u}+\varepsilon$. Since $\varphi_{1}(u)$ and $\varphi_{2}(u)$ are the solutions of Eq. (8),

$$
\left\|A \varphi_{1}-A \varphi_{2}\right\|=|| \varphi_{1}-\varphi_{2} \|=\lim _{k \rightarrow \infty}\left|\varphi_{1}\left(u^{k}\right)-\varphi_{2}\left(u^{k}\right)\right| .
$$

The following estimates are true for sufficiently large $k$ :

$$
\begin{gathered}
\left|A \varphi_{1}\left(u^{k}\right)-A \varphi_{2}\left(u^{k}\right)\right| \leq p_{0}\left|\varphi_{1}\left(u^{k}+c\right)-\varphi_{2}\left(u^{k}+c\right)\right| \\
+\sum_{z_{n} \leq u / v} p_{n}\left|\varphi_{1}\left(u^{k}-z_{n} v\right)-\varphi_{2}\left(u^{k}-z_{n} v\right)\right| \leq p_{0}\left(|| \varphi_{1}-\varphi_{2}||-\delta\right)+|| \varphi_{1}-\varphi_{2}\left\|\sum_{z_{n} \leq u / v} p_{n} \leq|| \varphi_{1}-\varphi_{2}\right\|-p_{0} \delta .
\end{gathered}
$$

Passing here to the limit in $k$, we obtain an inconsistency:

$$
\lim _{k \rightarrow \infty}\left|A \varphi_{1}\left(u^{k}\right)-A \varphi_{2}\left(u^{k}\right)\right|=\left\|\varphi_{1}-\varphi_{2}\right\| \leq\left\|\varphi_{1}-\varphi_{2}\right\|-p_{0} \delta
$$

The theorem is proved.

THEOREM 2 (convergence of the method of successive approximations for the solution of Eq. (8)). With the condition (11) for any initial approximation $\varphi^{0}(u)$ such that $\varphi_{*}(u) \leq \varphi^{0}(u) \leq 1$, the sequence of approximations $\varphi^{k+1}(u)=A \varphi^{k}(u), k=0,1, \ldots$ (where the operator $A$ is defined by the right-hand side of Eq. (8)) converges pointwise to the monotonic solution $\varphi(u)$ of Eq. (8), continuous on the right, such that $\varphi_{*}(u) \leq \varphi(u) \leq 1$.

Proof. Denote by $\left\{\bar{\varphi}^{k}(u)=A \bar{\varphi}^{k-1}(u), \bar{\varphi}^{0}(u) \equiv 1, k=0,1, \ldots\right\}$ a monotonically deceasing sequence of approximations that starts with unity, and by $\left\{\underline{\varphi}^{k}(u)=A \underline{\varphi}^{k-1}(u), \underline{\varphi}^{0}(u)=\varphi_{*}(u), k=0,1, \ldots\right\}$ a monotonically increasing sequence of 


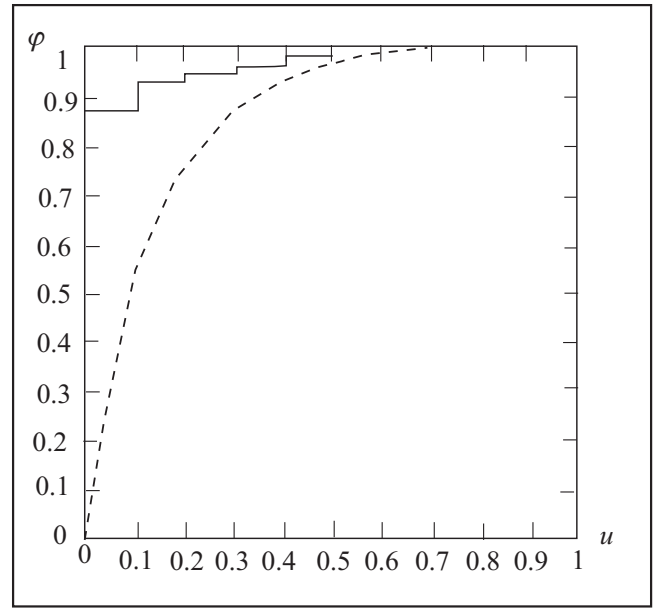

Fig. 1. Probability of retaining investments as a function of the initial insurance reserve.

approximations that starts with $\varphi_{*}(u)$. Both sequences converge pointwise to a unique (according to Theorem 1) monotonic solution $\varphi(u)$ of Eq. (8), continuous on the right. Since $\varphi_{*}(u) \leq \varphi^{0}(u) \leq 1$ and the operator $A$ is monotonic for all $k$, $\varphi_{*}(u) \leq \underline{\varphi}^{k}(u) \leq \varphi^{k}(u) \leq \bar{\varphi}^{k}(u) \leq 1$. Therefore, the sequence of monotonic functions $\left\{\varphi^{k}(u)\right\}$ also converges pointwise to the solution $\varphi(u)$ of Eq. (8). The theorem is proved.

Example. Let investments in an object of cost $v=1$ bring in a return of $20 \%$, half of which goes into the insurance fund, i.e., $c / v=0.1$. Let also relative loss constitute $z=(0.1 ; 0.3 ; 0.5 ; 0.7)$ for catastrophic events, which may happen every year with probabilities $p=(0.05 ; 0.01 ; 0.005 ; 0.001)$, respectively. The probability that no catastrophic event will happen during the year is equal to $p_{0}=1-0.066=0.934$. Then condition $(11) c=0.1>\frac{v}{p_{0}} \sum_{n=1}^{N} p_{n} z_{n}=0.012$ holds. The root of Eq.

(10) is equal to $\alpha=7.2939$. The dashed line in Fig. 1 shows the lower bound for the solution of Eq. (8), which has the form $\varphi_{*}(u)=1-e^{-7.2939 u}$ in the example, and the solid line shows the solution of Eq. (8) obtained in 20 iterations by the method of successive approximations with the accuracy 0.001 .

\section{REFERENCES}

1. Yu. M. Ermol'yev, T. Yu. Ermol'yeva, G. McDonald, and V. I. Norkin, "Problems of insurances of catastrophic risks," Cybern. Syst. Analysis, 37, No. 2, 220-234 (2001).

2. Z. Body, A. Kane, and A. Markus, Essentials of Investments, 4th ed., McGraw-Hill (2001).

3. R. T. Rockafellar and S. Uryasev, "Optimization of conditional value-at-risk," J. Risk, 2, $21-42$ (2000).

4. V. I. Norkin, “On measuring and profiling catastrophic risks,” Cybern. Syst. Analysis, 42, No. 6, 839-850 (2006).

5. M. M. Leonenko, Yu. S. Mishura, Ya. M. Parkhomenko, and M. J. Yadrenko, Probability-Theoretic and Statistical Methods in Econometrics and Financial Mathematics [in Ukrainian], Informtekhnika, Kyiv (1995).

6. R. E. Beard, T. Pentikäinen, and E. Pesonen, Risk Theory. The Stochastic Basis of Insurance, 3rd ed., Chapman and Hall, London-New York (1984).

7. S. Asmussen, Ruin Probabilities, World Scientific, Singapore (2000).

8. B. V. Norkin, "Calculating ruin probability of a non-Poisson risk process by the method of successive approximations," Probl. Upravl. Inform., No. 2, 133-144 (2005).

9. A. V. Boikov, "The Cramer-Lundberg model with stochastic premiums," Teor. Veroyan. i yeyo Primen., 47, Issue 3, 549-553 (2002).

10. B. V. Norkin, "The method of successive approximations applied to find the nonbankruptcy probability for an insurance company with random premiums," Cybern. Syst. Analysis, 42, No. 1, 98-110 (2006).

11. V. I. Norkin, "Solving the Wiener-Hopf equation with a probabilistic kernel," Cybern. Syst. Analysis, 42, No. 2, 195-201 (2006). 